\title{
Design of an Ultra-High Resolution SEM for Enhanced Analysis
}

\author{
Jaroslav Jiruše ${ }^{1}$, Miloslav Havelka ${ }^{1}$ and Jan Polster ${ }^{1}$ \\ 1. TESCAN Brno, Brno, Czech Republic
}

Along with the recent advances in nanotechnology there is a growing need for a SEM combining ultra-high resolution optics and analytical performance. Here we introduce a new design of a SEM column where recently-developed high-resolution optics [1] is brought together with traditional analytical capabilities.

The column is equipped with a well-known single-pole objective lens [2] that creates a strong magnetic field around the sample, thus dramatically decreasing optical aberrations. Design optimization and combining the magnetic immersion field with the electrostatic field [3] further improves the resolution to less than $1.1 \mathrm{~nm}$ at $1 \mathrm{kV}$. For analytical purposes (EDS or EBSD) or for use with a FIB column however, the immersion magnetic field becomes a complication due either to curvature of signal electron trajectories or beam splitting of ion-isotopes.

A magnetic-field-free mode (in the vicinity of the specimen) with an intermediate lens is commonly used, but the resolution is quite poor. To overcome this, we have redesigned the objective system adding a third objective lens closer to the sample, which provides a resolution of $2.5 \mathrm{~nm}$ in the magnetic-field-free mode.

The triple-objective lens design expands the set of display modes and allows a new analytical mode for FIB processing and analytical tasks. Moreover, the redesigned electron gun allows to reach a current as high as $400 \mathrm{nA}$ for both structural analysis and ten times faster beam energy alternation. This geometry of the objective also allows processing of 8 inch wafers.

To achieve high thermal stability with respect to the immersion lens with high values of coils excitation, the column works in a regime where constant thermal power dissipation is maintained independent of excitation. Thus, time-consuming automatic procedures can be performed. For example, 3D BSE tomography of an SERS active structure (gold-coated partially etched polystyrene spheres - Figure 1) took 13 hours [4]. The sample was sliced by the FIB and each cross-section was imaged at $2 \mathrm{kV}$.

The column geometry is optimized for this detection system. Two triplets of detectors are dedicated for each type of signal electrons, comprising secondary electrons (SE) and back-scattered electrons (BSE). Thereby BSEs with different take-off angles can be captured by three distinct detectors, one in the chamber and two in the column. Moreover, the BSE detectors equipped with a biased grid enable energy filtration to get a new surface-sensitive signal with enhanced material contrast, see Figure 2.

\section{References:}

[1] J Jiruse et al, Microsc. Microanal. 19 (Suppl 2), 2013, p. 1302.

[2] Z Shao, PSD Lin, Rev. Sci. Instrum. 60(11), 1989, p. 3434.

[3] J Jiruše et al, Ultramicroscopy 146, 27 (2014)

[4] L Stolcova et al, Imaging \& Microscopy, Issue 4, 2015, p. 34. 


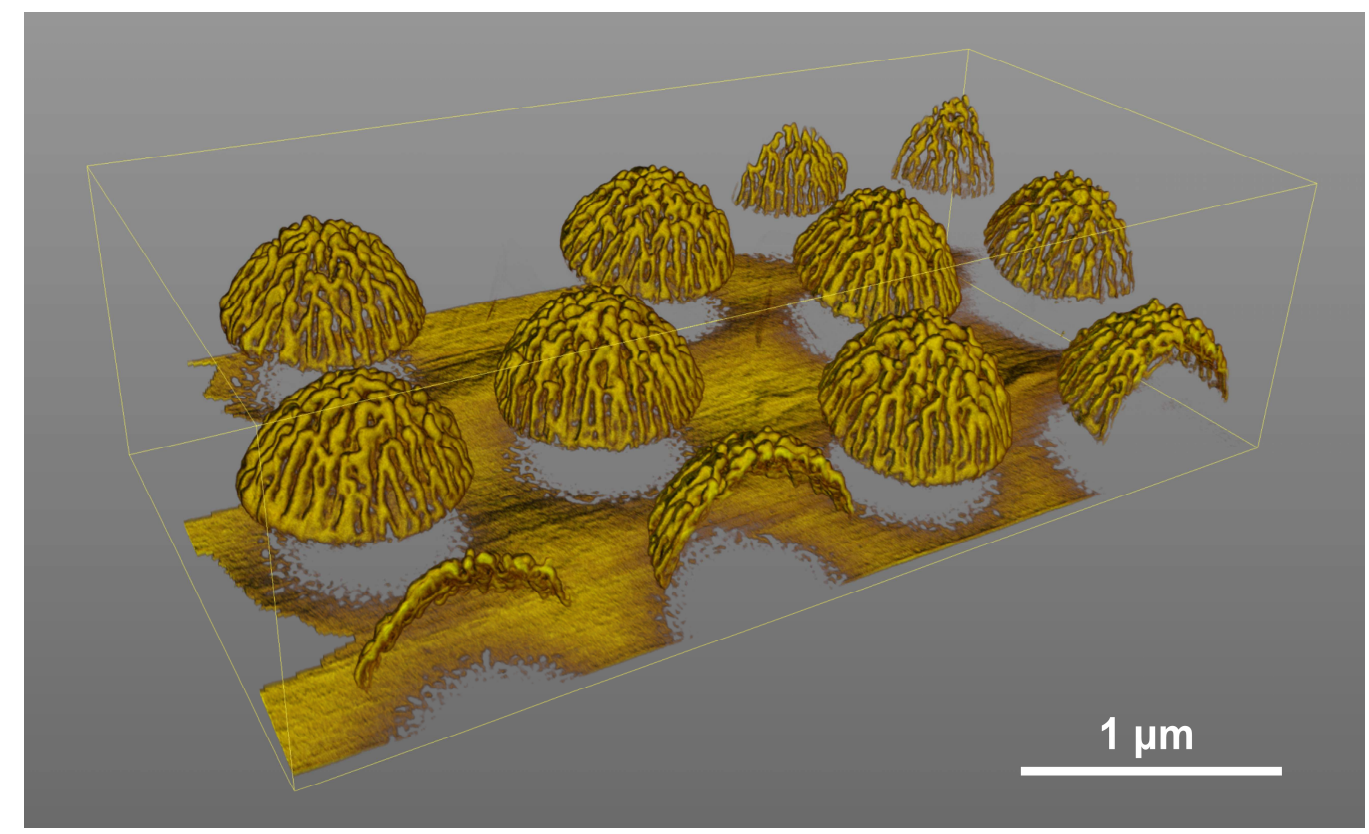

Figure 1. 3D BSE reconstruction of a SERS active structure (gold-coated partially etched polystyrene spheres) at $2 \mathrm{keV}$. Two hundred slices were acquired in 13 hours with a resolution of $3 \times 3 \times 10 \mathrm{~nm}$.
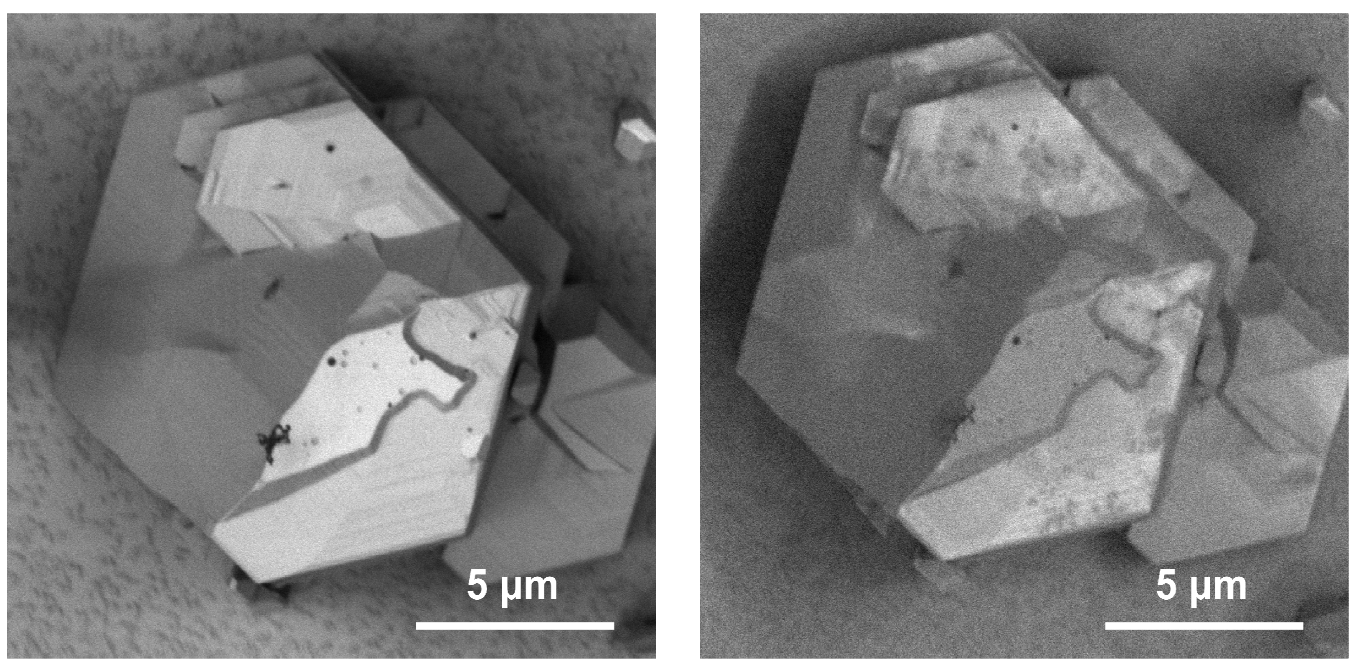

Figure 2. BSE images of GaN. Left: standard BSE signal at $2 \mathrm{keV}$. Right: Filtered image, obtained by capturing low-loss BSE in the energy window from $1.95 \mathrm{keV}$ to $2 \mathrm{keV}$, provides more surface-sensitive signal and less topographic information. 